\title{
Application of the modified compaction material model to the analysis of landmine detonation in soil with various degrees of water saturation
}

\author{
M. Grujicic a,*, B. Pandurangan ${ }^{\text {a }}$, J.D. Summers ${ }^{\text {a }}$, B.A. Cheeseman ${ }^{\text {b }}$, W.N. Roy ${ }^{\mathrm{b}}$ and R.R. Skaggs $^{\mathrm{c}}$ \\ ${ }^{a}$ Department of Mechanical Engineering, Clemson University, Clemson SC 29634, USA \\ ${ }^{\mathrm{b}}$ Army Research Laboratory - Survivability Materials Branch, Aberdeen, Proving Ground, MD 21005-5069, USA \\ ${ }^{\mathrm{c}}$ Army Research Laboratory - Explosive Technologies Branch, Aberdeen, Proving Ground, MD 21005-5069, USA
}

Received 24 July 2006

Revised 4 January 2007

\begin{abstract}
A series of transient non-linear dynamics computational analyses of the explosion phenomena accompanying the detonation of a $100 \mathrm{~g} \mathrm{C} 4$ mine buried in sand to different depths is carried out using the software package AUTODYN. The mechanical response of sand under high deformation-rate conditions has been represented using the modified compaction material model developed in our recent work [1]. While the mechanical response of the other attendant materials (air, gaseous-detonation products and AISI 1006 mild steel) is accounted for using the material models available in literature. The results obtained (specifically, the temporal evolution of the sand overburden shape and pressure at various locations in air above the detonation site) were compared with their experimental counterparts for a $(50 \mathrm{wt} \%$-sand $/ 50 \mathrm{wt}$.\%-clay) soil obtained recently by Foedinger [2]. The comparison revealed that the modified compaction material model for sand can account reasonably well for the magnitude, spatial distribution and the temporal evolution of the dynamic loads accompanying detonation of shallow-buried mines in soils with various clay and water contents.
\end{abstract}

Keywords: Detonation, shallow buried mine, blast loading, AUTODYN

\section{Nomenclature}

$A-\quad$ Room temperature yield stress

$A_{1}-$ Constant in JWL Equation of State

$\alpha-\quad$ Porosity

$B$ - Compaction Modulus

$B_{1}-$ Strain Hardening Constant

$B_{2}-$ Constant in JWL Equation of State

\footnotetext{
${ }^{*}$ Corresponding author: Mica Grujicic, 241 Engineering Innovation Building, Clemson University, Clemson, SC 29634-0921, USA. Tel.: +1 864656 5639; Fax: +1 864656 4435; E-mail: mica.grujicic@ces.clemson.edu.
} 
$\beta-\quad$ Saturation ratio

$C-\quad$ Speed of sound

$C_{1}-$ Strain rate constant

$E-\quad$ Internal energy

$\varepsilon-\quad$ Plastic strain

$G-\quad$ Shear modulus

$\gamma-\quad$ Constant-Pressure to constant-volume specific heats ratio

$\gamma_{1}-$ Saturation Parameter

$\Gamma-\quad$ Gruneisen parameter

$K-\quad$ Bulk Modulus

$\mu-\quad$ Compression ratio

$\mu_{1}-\quad$ Yield-Stress-to-Pressure Proportionality Coefficient

$P$ - Pressure

$R_{1}-$ Constant in JWL Equation of State

$R_{2}-$ Constant in JWL Equation of State

$\sigma-\quad$ Yield Stress

$T-\quad$ Temperature

$v-\quad$ Specific volume

$w-\quad$ Constant in JWL Equation of State

$x-\quad$ Spatial coordinate

$y$ - Spatial coordinate

$Y-\quad$ Yield stress

\section{Subscripts}

$\begin{array}{ll}\text { Comp - } & \text { Value at full compaction } \\ \text { dry }- & \text { Dry Sand quantity } \\ \text { melt }- & \text { Melting point quantity } \\ o- & \text { Initial value } \\ p- & \text { Pore related quantity } \\ \mathrm{pl}- & \text { Plastic state quantity } \\ \text { Pl.Comp - } & \text { Plastic Compaction related quantity } \\ \text { room - } & \text { Room temperature quantity } \\ s- & \text { Fully-compacted sand related quantity } \\ \text { Solid.Comp }- & \text { Solid Compaction related quantity } \\ H- & \text { Homologous quantity } \\ \text { sat }- & \text { Saturation related quantity } \\ \text { Unsat }- & \text { Unsaturated Sand related quantity } \\ w- & \text { Water related quantit }\end{array}$

\section{Superscripts}

* - Value at minimum pressure for full sand compaction

$m-\quad$ Thermal softening exponent

$n-\quad$ Strain hardening exponent

\section{Introduction}

It is well established that buried and ground-laid landmines represent a major threat to lightweight vehicles like the High Mobility Multi-Purpose Military Vehicles [3]. The observed destruction arising from detonation of the 


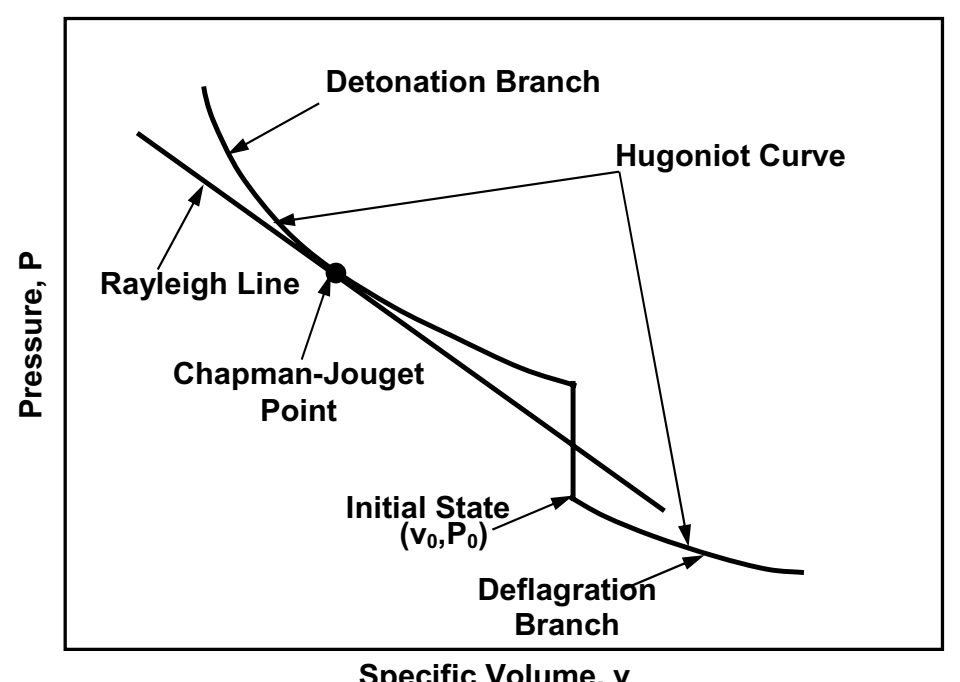

Fig. 1. Pressure vs. specific volume relations in a high-energy explosive material. Please see the text for details.

landmines is the result of the large impulsive loads brought about by blast and shock waves, mine fragments and soil ejecta. The design of survivable vehicles and platforms (targets) requires the ability to understand and quantify the impulsive detonation loads from the landmines buried in different soil media and to model the ballistic, structural and thermal response of structures/targets (e.g. vehicles, platforms, etc.) of interest. Quantification and elucidation of the (time-dependent) load a buried landmine applies to a target structure above it when the landmine is detonated is quite challenging since such load depends on the size and shape of the charge, its depth of burial, the distance between the soil surface and the target, and the properties (density, particle size and distribution, presence of organic matter, water content, etc.) of the soil in which the landmine is buried. While the direct experimental characterizations of landmine-blast events are highly critical for obtaining a better understanding of the accompanying complex phenomena, this approach is not practical and often cost prohibitive. Recent developments in the numerical modeling of transient non-linear dynamics phenomena, in particular, the ability to couple Eulerian solvers (used to model gaseous detonation products and air) and Lagrangian solvers (used to represent vehicles/platforms and soil), have allowed simulations to provide an additional insight into complex loading created by the mine blast event. However, the fidelity of the computational models remains an outstanding issue. As discussed in our previous work [1], the lack of fidelity of computer simulations of the blast event is mainly due to inability of the currently available material model to realistically represent the response of the materials involved under high-deformation, high deformationrate high-temperature conditions, the type of conditions accompanying landmine detonation. In particular, the soil response and its dependence on the soil composition, microstructure and water content are poorly understood [3]. In addition, a better understanding of the ballistic and structural response of the welded joints used in the construction of vehicles and platforms is needed.

A review of the literature shows that there exists an extensive body of work dealing with the investigation of the detonation of the buried charges. However, much of this work does not focus on the characterization of the blast output of landmines, but rather on cratering effects in soils, with applications towards the efficient utilization of explosives for excavation (i.e. canals, trenches, etc.) or in the survivability of structures subjected to near surface blasts [4]. Among the work published in the open literature which directly deal with experimental characterization of the effects of anti-tank (AT) and anti-personnel (AP) landmine blasts, the following appear to be the most relevant to the present subject matter. Westine et al. [5] carried out experiments on a plate which was mounted above a buried charge comparable is size and power to an AT landmine. The plate contained a number of through-the-thickness holes at incremental distances from the mine, in which, plugs of known mass were placed. The blast accompanying mine detonation caused the plugs to be ejected from the holes and from their initial velocity the impulsive loading on the plate was calculated. Morris [6] used the results of Westine et al. [5] to construct a design-for-survivability computer code for lightweight vehicles. More recently, Bergeron et al. [7] carried out a comprehensive investigation 


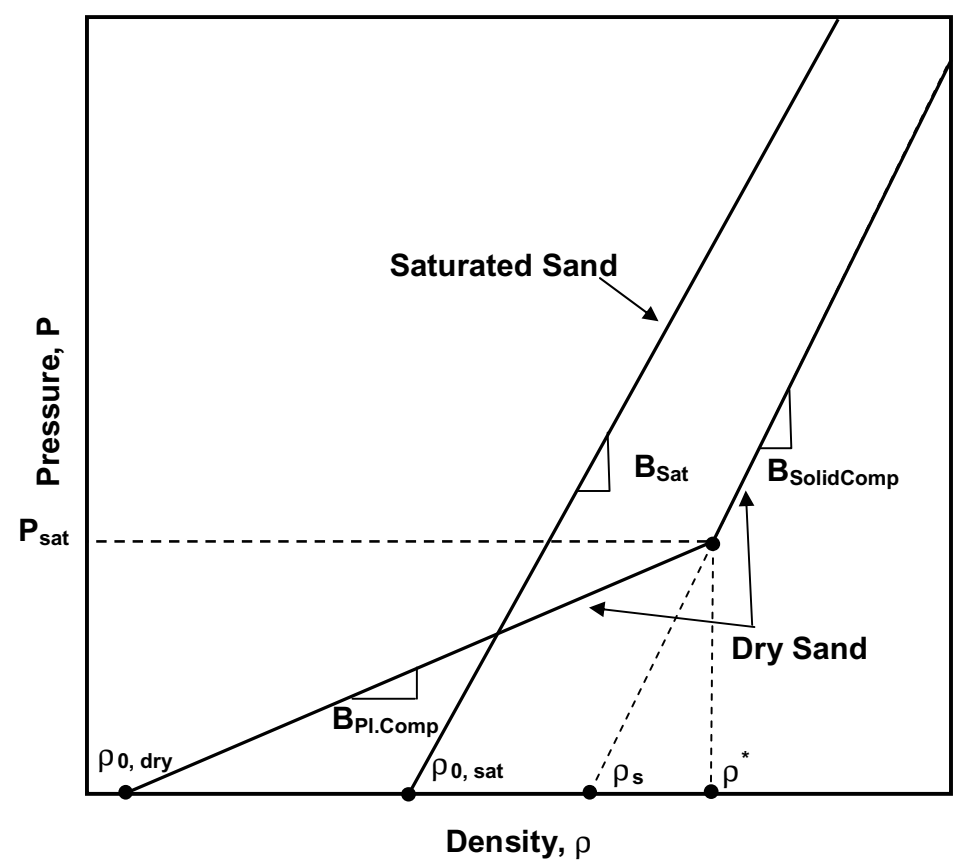

Fig. 2. A schematic of pressure vs. density relations for the dry and saturated sand.

of the buried landmine blasts using an instrumented ballistic pendulum. From these experiments, the pressure and impulse as a function of time were recorded at several locations in air directly above the mine as well as in the sand surrounding the mine, along with $\mathrm{x}$-radiographs and high speed photographs of the associated soil cratering and ejecting phenomena. The work of Bergeron et al. [7] was subsequently extended by Braid [8] to incorporate different charge sizes, soil types and improved instrumentation.

In our recent computational work [9], based on the use of AUTODYN, a general-purpose transient non-linear dynamics explicit simulation software [10], a detailed comparison was made between the experimental results of Bergeron et al. [11] and their computational counterparts for a number of detonation-related phenomena such as the temporal evolutions of the shape and size of the over-burden sand bubbles and of the detonation-products gas clouds, the temporal evolutions of the side-on pressures in the sand and in air, etc. It was found that the most critical factor hampering a better agreement between the experiment and computational analysis is an inadequacy of the current material model for sand to capture the dynamic response of this material under blast loading conditions. Hence, the main objective of our subsequent work [1] was to improve the compaction material model for sand in order to include the effects of the degree of saturation and rate of deformation, the two important effects which were neglected in the original compaction model (proposed by Laine and Sandvik [20]) used in AUTODYN [10]. The new material constitutive model for sand was subsequently validated for the case of sand with different levels of (water) saturation by comparing he experimental results associated with detonation of the shallow-buried and ground-laid C4 mines obtained through the use of an instrumented horizontal mine-impulse pendulum with their computational counterparts obtained via detailed numerical modeling of the same physical problem using AUTODYN. In our subsequent work [12], the ability of the newly developed material model to predict the temporal evolutions of the blast loads associated with the detonation of mines buried in fully water-saturated sand was tested. This was done by comparing the model predictions with their experimental counterparts obtained in the work of Taylor and Skaggs [13] who carried out large-scale experiments using the Vertical Impulse Measurement Fixture (VIMF) at the Army Research Laboratory, Aberdeen, MD. The VIMF is a unique facility that has been designed specifically to measure accurately the vertical impulse from buried charges weighing up to eight kilograms [13].

The two main objectives of the present work are: (a) to simplify our recently developed compaction model for sand by eliminating the explicit strain-rate dependences of its parameters and to upgrade the model by introducing a degree of saturation dependent (negative) minimum failure pressure; and (b) to carry out a series of transient 
non-linear dynamics analyses of detonation of a $100 \mathrm{~g} \mathrm{C} 4$ mine buried to different depths in sand with varying levels of saturation and to compare the computed results with their experimental counter parts obtained recently by Foedinger [2] for a (50wt\%-sand/50wt.\%-clay) soil. Specifically, a comparison is made between the computed and the experimental results pertaining to the temporal evolution of the sand (soil) overburden shape and pressures in air at different locations above the explosion site. The extent of agreement between the computational and experimental results is then used to assess the need for further development of the sand compaction model in order to include inorganic additives/binders like clay.

The organization of the paper is as follows. An overview of the transient non-linear dynamics modeling of a land-mine detonation is presented in Section 2.1. The material constitutive models for the four materials used in the computational analysis and the definition of the computational problem investigated are respectively discussed in Sections 2.2 and 2.3. The results obtained in the present work are presented and discussed in Section 3. The main conclusions resulting from the present work are summarized in Section 4.

\section{Computational procedure}

\subsection{Transient non-linear dynamics modeling of the landmine detonation}

All the calculations carried out in the present work were done using AUTODYN, a general-purpose transient non-linear dynamics analysis software [10]. In our previous work [12], a detailed account was provided of the basic features of AUTODYN, emphasizing the ones which are most relevant for modeling detonation of shallow-buried and ground-laid mines. Therefore, only a brief overview of AUTODYN is given in this section.

A transient non-linear dynamics problem such as detonation of shallow-buried and ground-laid mines is analyzed within AUTODYN by solving simultaneously the governing partial differential equations for the conservation of momentum, mass and energy along with the material constitutive equations and the equations defining the initial and the boundary conditions. The aforementioned equations are solved numerically using a second-order explicit scheme. Due to the large motions and deformations of the attendant materials (air, sand and gaseous-detonation products), an Euler control-volume computational scheme is typically used when modeling mine detonation. Within the Euler scheme, a numerical grid is fixed in space while the material(s) move through it. In our previous work [12], a comprehensive discussion was given pertaining to how the governing conservation differential equations and material model constitute a self-consistent system of equations for the dependent variables. In particular, it was shown how the material constitutive models are coupled with the conservation differential equations.

Due to the fact that several materials may simultaneously reside within a single computational cell, the multimaterial Euler scheme of AUTODYN was used in the present work. Within this scheme, a control volume method is used to solve the integral and finite-difference forms of the mass, momentum and energy conservation equations in order to obtain an accurate and stable solution. The terms appearing in these equations are divided into two groups: Lagrangian and transport (convective). A two-step numerical procedure is used to solve the finite-difference form of the governing equations. Within the first step, the Lagrange (cell-deforming) step, the Lagrangian form of the governing equations is advanced one time interval. Within the second step, the Euler step, the dependant variables updated in the first step are mapped on to the un-deformed Euler mesh. Multiple materials are handled through a volume fraction technique or an interface technique developed by Young's [14]. All dependent variables are referenced with respect to the center of the cell.

It should be noted that while the Euler formulations are ideally suited for handling large deformations and fluid flow, they suffer from the difficulties in tracking free-surfaces, material interfaces and history-dependent material behavior. The Euler formulation may also be prone to numerical diffusion associated with material convection between cells. Numerous parallel investigations of the same problem using Lagrange and Euler processors revealed that the errors brought about by numerical diffusion are only in a range of few percent [10].

Since the material constitutive models play a dominant role in transient non-linear dynamics analysis like the one associated with mine-detonation, a detailed account of the constitutive models for the materials encountered in the present work is given in the next section. 


\subsection{Material constitutive models}

As discussed in the previous section, the complete definition of a transient non-linear dynamics problem entails the knowledge of the material model that define the relationships between the flow variables (pressure, mass-density, energy-density, temperature, etc.). These relations typically involve an equation of state, a strength equation and a failure equation for each constituent material. These equations arise from the fact that, in general, the total stress tensor can be decomposed into a sum of a hydrostatic stress (pressure) tensor (which causes a change in the volume/density of the material) and a deviatoric stress tensor (which is responsible for the shape change of the material). An equation of state then is used to define the corresponding functional relationship between pressure, mass density and internal energy density (temperature), while a strength relation is used to define the appropriate equivalent plastic strain, equivalent plastic strain rate, and temperature dependencies of the equivalent deviatoric stress. In addition, a material model generally includes a failure criterion, i.e. an equation describing the (hydrostatic or deviatoric) stress and/or strain condition(s) which, when attained, causes the material to fracture and lose its ability to support (abruptly in the case of brittle materials or gradually in the case of ductile materials) normal and shear stresses.

In the present work, the following four materials are utilized within the computational domain: air, AISI 1006 mild steel (the bucket), C4 (high-energy explosive) and sand. The various material models used for air, AISI 1006 mild steel (the bucket) and C4 (high-energy explosive) are provided in Table 3. The values of all the material parameters for air, AISI 1006 mild steel and C4 are available in the AUTODYN materials library [10] and, hence, can be accessed by all licensed AUTODYN users. A description of the material model for sand is given in the next section. The material model parameters for sand can be found in our previous work [1].

\subsubsection{Sand}

\subsubsection{Sand behavior under high deformation rates}

In general, sand has a complex structure consisting of mineral solid particles which form a skeleton. The pores between the solid particles are filled with air containing different extents of moisture. The sand containing air with less than ca. $15 \mathrm{wt}$.\% moisture is typically referred to as "dry sand", while the sand containing air with in excess of $90 \mathrm{wt} . \%$ moisture content is referred to as " saturated sand". The relative volume fractions of the three constituent materials in the sand (the solid mineral particles, water and air) are generally quantified by the porosity, $\alpha$, and the degree of (water) saturation (Saturation Ratio), $\beta$, which are respectively defined as:

$$
\alpha=\frac{V_{p}}{V}
$$

and

$$
\beta=\frac{V_{w}}{V_{p}}
$$

where $V_{p}$ is the volume of void (pores), $V_{w}$ the volume of water and $V$ the total volume.

Sand-particle surface roughness and the presence of inorganic/organic binders within sand pores are generally considered to be the main causes for friction and adhesion at the inter-particle contacting surfaces. Deformation of the sand is generally believed to involve two main basic mechanisms [18,19]: (a) reversible deformations (at low pressure levels) and fracture (at high pressure levels) of the inter-particle bonds and (b) elastic and plastic deformations of the three constituent materials (solid mineral particles, water and air) as well as fracture of the solid mineral particles in the sand. The relative contributions of these two deformation mechanisms as well as their behavior are affected primarily by the degree of saturation of sand and the deformation rate. Specifically, in dry sand the first mechanism controls the sand deformation at low pressures while the second mechanism is dominant at high pressures and the effect of deformation rate is of a second order. In sharp contrast, in saturated sand very low inter-particle friction diminishes the role of the first deformation mechanism. On the other hand, the rate of deformation plays an important role. At low deformation rates, the water/air residing in the sand pores is readily squeezed out during deformation and, consequently, the deformation of the sand is controlled by the deformation of the solid mineral particles. At high pressures, on the other hand, water/air is trapped within the sand pores and the deformation of the sand is controlled by the deformation and the volume fractions of each of the three constituent phases. 


\subsubsection{Compaction material model for sand and its recent modifications}

Within AUTODYN, the dynamic response of sand is represented using a compaction material model which was formulated using the experimental results obtained by Laine and Sandvik [20]. A detailed description of the original compaction material model for sand was given in our recent work. In the same work [1], a set of modifications was proposed to the original compaction model to include the effects of deformation rate and the degree of saturation on the mechanical constituent response of the sand. The modifications of the original compaction model carried out in our previous work [1], involved changes in the equation of state and the strength model. The modifications of the equation of state were based on the following set of assumptions:

(a) The average sand particle size, particle size distribution and the presence of inorganic/organic natural matter in sand all have a second order effect on the dynamic constituent response of the sand. This assumption was justified by the experimental observations reported in Ref. [3] which clearly showed that the effect of sand type (e.g. prairie sand containing high level of silt and clay, impurity-free $-30 /+50$ sand, etc.), on the detonation-induced momentum transfer to the instrumented horizontal mine-impulse pendulum was small in comparison with the effect of the degree of saturation;

(b) The dynamic mechanical response of the sand at any degree of saturation can be obtained as a linear combination of the corresponding dynamic material behaviors for the dry and the saturated sand;

(c) The dynamic mechanical response of the dry sand is not rate dependent and it can be represented by the original compaction model implemented in AUTODYN [10]. It should be noted that Jackson et al. [23] observed that dry sand displays a rate-dependent behavior. However, the rate dependency of dry sand is still quite small in comparison to that of saturated sand and, hence was ignored in the present work;

(d) The initial density of the saturated sand, $\rho_{\mathrm{o} \text {,sat }}$, can be calculated using densities of the solid material in the sand, $\rho_{s}$, and water, $\rho_{w}$, and the known level of sand initial porosity, $\alpha_{o}=1-\left(\rho_{\mathrm{o}, \mathrm{dry}} / \rho_{s}\right)$, as:

$$
\rho_{\mathrm{o}, \mathrm{sat}}=\rho_{s}\left(1-\alpha_{o}\right)+\rho_{w} \alpha_{o}
$$

(e) When the saturated sand is subjected to relatively low deformation (compression) rates, water is given enough time to leave the pores and hence the density of the fully compacted sand and the pressure at which full compaction is attained are identical to their counterparts in the dry sand [24];

(f) When the saturated sand is subjected to very high compression rates, the water becomes trapped inside the sand pores and, under such circumstances, the saturated sand behaves as a fully compacted sand and can undergo only an elastic compaction. Due to significantly lower compressibility of the water and the fact that both water and solid sand-particles form continuous networks, the compressibility of saturated sand is controlled by the compressibility of its solid phase; and

(g) Under intermediate deformation rates, the dynamic material response of the saturated sand can be obtained using a linear interpolation of the high- and low-deformation rate behaviors of the saturated sand. A value of $1.0 \cdot 10^{5} \mathrm{~s}^{-1}$ is used as the "high" deformation rate, $\dot{\varepsilon}_{\text {high }}$, and a value of $1.0 \cdot 10^{-3} \mathrm{~s}^{-1}$ is used as the "low" deformation rate, $\dot{\varepsilon}_{\text {low }} \cdot \dot{\varepsilon}_{\text {low }}$. In our previous work [1], it was shown that the computational results are essentially insensitive to a one order of - magnitude increase/decrease of the lower and the upper deformation rates. At the deformation rates exceeding $1.0 \cdot 10^{5} \mathrm{~s}^{-1}$ and at the deformation rates below $1.0 \cdot 10^{-3} \mathrm{~s}^{-1}$, the dynamic behavior of sand is assumed to be rate independent and to correspond to the dynamic sand behavior at the respective $\left(1.0 \cdot 10^{5} \mathrm{~s}^{-1}\right.$ or $\left.1.0 \cdot 10^{-3} \mathrm{~s}^{-1}\right)$ deformation rates. In the absence of relevant experimental data, a phenomenological approach was adopted according to which the strain rate dependency of material density at the intermediate deformation rates is defined using a linear interpolation procedure based on the logarithms of the deformation rates as:

$$
\rho=\rho_{\text {high }}+\left(\rho_{\text {low }}-\rho_{\text {high }}\right)\left(\frac{\log \dot{\varepsilon}-\log \dot{\varepsilon}_{\text {high }}}{\log \dot{\varepsilon}_{\text {low }}-\log \dot{\varepsilon}_{\text {high }}}\right)
$$

where the densities $\rho, \rho_{\text {high }}$ and $\rho_{\text {low }}$ correspond respectively to the deformation rates $\dot{\varepsilon}, \dot{\varepsilon}_{\text {high }}$ and $\dot{\varepsilon}_{\text {low }}$ and are all associated with the same level of pressure. The computational results obtained are found not to be significantly affected by an order of magnitude changes in the values for the high and low deformation rates.

The modifications of the original strength compaction model for sand to include the inter-particle water-induced lubrication effect as carried out in our recent work [12] was based on the following set of assumptions: 
Table 1

Parameters appearing in the definition of simplified modified material model for dry, unsaturated and saturated sand

\begin{tabular}{llll}
\hline Parameter & Symbol & Unit & Value \\
\hline Density of solid sand particles & $\rho_{s}$ & $\mathrm{~kg} / \mathrm{m}^{3}$ & 2641 \\
Density of water & $\rho_{w}$ & $\mathrm{~kg} / \mathrm{m}^{3}$ & 1000 \\
Solid sand compaction modulus & $B_{\text {SolidComp }}$ & $\mathrm{MPam}^{3} \mathrm{~kg}^{-1}$ & 21.68 \\
Bulk modulus of water & $B_{w}$ & $\mathrm{MPam}^{3} \mathrm{~kg}^{-1}$ & 2.150 \\
Minimum pressure for full compaction of dry sand & $P_{\text {Comp }}$ & $\mathrm{GPa}$ & 0.6506 \\
\hline
\end{tabular}

(a) The "compaction" strength model for sand is based on an isotropic, perfectly plastic, rate independent yieldsurface approximation and postulates that the material's yield strength depends explicitly on pressure and not on material density. The yield stress quantifies the resistance of the material to a plastic (irreversible) shape change. The plastic shape change occurs when the magnitude of the second invariant of the deviatoric part of the stress tensor becomes equal to the yield stress;

(b) Unloading (and subsequent reloading) of a previously plastically deformed material is of an elastic (reversible) nature and, in this case, the deviatoric stress is proportional to the deviatoric strain with the proportionality constant being equal to the shear modulus, $G$. Due to the porous nature of sand, its shear modulus is taken to depend on its mass-density;

(c) The experimental results of Laine and Sandvik [20] revealed that the values of the ratio of the yield stress to the pressure (which scales with the effective inter-particle friction coefficient) falls in a narrow range between 1.35 and 1.40 for dry sand;

(d) The presence of water in saturated sand creates a water-based shear layer physically separating sand particles and reduces the effective friction coefficient and, thus the yield stress;

(e) While the thickness of such a layer (and thus the magnitude of the inter-particle friction coefficient) is dependent on the rate of deformation, only pressure-dependent values of the inter-particle friction coefficient in the limit of infinite deformation rates of the saturated sand are used; and

(f) Following the work of Dowson and Ehret [21], the values of the inter-particle friction coefficient in the saturated sand are set to vary between 0.1 at the lowest pressures and 0.8 at the highest pressures. In accordance with the von Mises yield criteria, the yield stress at a given pressure level is defined as the square root of 3 multiplied by a product of the friction coefficient and the pressure.

The failure behavior of sand is modeled within the AUTODYN materials database by specifying a minimum (negative) value of the hydrodynamic pressure below which, the material fractures, and looses its ability to support any tensile or shear stresses. However, if a given "fractured" sand region is subsequently subjected to positive pressures, it is allowed to re-heal by closing up its cracks and to regain an ability to support shear stresses. A zero value of the failure hydrodynamic pressure was used in our prior work [20]. In other words, no modifications in the original failure compaction model for sand were made.

The application of the revised compaction model for sand carried out in Ref. [1], revealed that the modified compaction model for sand can be simplified by making it deformation-rate independent, provided high deformationrate values for the material model parameters are used. In the next section, a detailed procedure is presented for simplification of the modified compaction model for sand.

\subsubsection{Simplification of the modified compaction material model for sand}

In this section, simplifications are presented for the equation of state and the strength equation for the modified sand compaction material model. In addition, a modified failure model for sand is proposed. Table 1 contains a list of all parameters appearing in the definition of the simplified modified material model for dry, unsaturated and saturated sand.

Equation of State

The pressure vs. density behavior of dry sand, as discussed earlier, is represented using the original compaction model in the form:

$$
P_{\text {dry }}= \begin{cases}0 & \rho_{\text {dry }} \leqslant \rho_{\mathrm{o}, \text { dry }} \\ B_{\text {Pl.Comp }}\left(\rho_{\text {dry }}-\rho_{\mathrm{o}, \text { dry }}\right) & \rho_{\mathrm{o}, \text { dry }} \leqslant \rho_{\text {dry }} \leqslant \rho_{\text {dry }}^{*} \\ B_{\text {SolidComp }}\left(\rho_{\text {dry }}-\rho_{s}\right) & \rho_{\text {dry }}>\rho_{\text {dry }}^{*}\end{cases}
$$


where $B_{\text {Pl.Comp }}$ and $B_{\text {SolidComp }}\left(=21.68 \mathrm{MPa} . \mathrm{m}^{3} / \mathrm{kg}\right.$ ) are respectively the plastic compaction (densification) and the solid-particle compaction moduli, while $\rho_{\mathrm{o}, \mathrm{dry}}=\left(1-\alpha_{o}\right) \rho_{s}$ and $\rho_{s}\left(=2641 \mathrm{~kg} / \mathrm{m}^{3}\right)$ are the initial density of dry sand and the density of the fully compacted sand, respectively and $\alpha_{o}$ denotes the initial porosity in sand. It should be noted, that the compaction moduli used in Eq. (5) are defined as a ratio of the corresponding bulk moduli and mass-densities. The plastic compaction modulus, $B_{\mathrm{Pl} \text {.Comp }}$, is defined as:

$$
B_{\mathrm{Pl} . \text { Comp }}=\frac{P_{\mathrm{Comp}}}{\left(\rho_{\mathrm{dry}}^{*}-\rho_{\mathrm{o}, \mathrm{dry}}\right)}
$$

where $P_{\mathrm{Comp}}(=0.6506 \mathrm{GPa})$ is the minimum pressure needed for full densification of sand and $\rho_{\mathrm{dry}}^{*}$ is given by;

$$
\rho_{\text {dry }}^{*}=\rho_{s}+\frac{P_{\text {Comp }}}{B_{\text {SolidComp }}}
$$

The pressure vs. density curve for saturated sand is taken to be rate independent and to correspond to the $P$ vs. $\rho$ relationship associated with a (high) deformation rate. The relationship can be expressed as:

$$
P_{\text {sat }}= \begin{cases}0 & \rho_{\text {sat }} \leqslant \rho_{\text {o,sat }} \\ B_{\text {Sat }}\left(\rho_{\text {sat }}-\rho_{\mathrm{o}, \text { sat }}\right) & \rho_{\text {sat }}>\rho_{\mathrm{o}, \text { sat }}\end{cases}
$$

where $B_{\text {Sat }}$ is the compaction modulus of saturated sand and is defined using the compaction modulus of solid particles, $B_{\text {SolidComp }}$ and the compaction modulus of water, $B_{w}$, and the fact that both the solid phase and the water-filled porosity form continuous networks, as:

$$
B_{\text {Sat }}=(1-\alpha) B_{\text {SolidComp }}+\alpha B_{w}
$$

while $\rho_{\mathrm{o} \text {,sat }}$ is the initial density of saturated sand and is defined in terms of the density of solid mineral particles, $\rho_{s}$, and the density of water, $\rho_{w}$, as:

$$
\rho_{\mathrm{o}, \mathrm{sat}}=\left(1-\alpha_{o}\right) \rho_{s}+\alpha_{o} \rho_{w}
$$

The pressure vs. density curve for unsaturated sand is obtained as a linear combination of the pressure vs. density relations for the dry and the saturated sands, as:

$$
P_{\text {unsat }}\left(\alpha_{o}, \beta\right)= \begin{cases}0 & \rho_{\text {unsat }} \leqslant \rho_{\text {o, unsat }} \\ B_{\text {unsat low }}\left(\rho_{\text {unsat }}-\rho_{\text {o,unsat }}\right) & \rho_{\text {o,unsat }} \leqslant \rho_{\text {unsat }} \leqslant \rho_{\text {unsat }}^{*} \\ B_{\text {unsat,high }}\left(\rho_{\text {unsat }}-\rho_{\text {unsat }}^{*}\right) & \rho_{\text {unsat }}>\rho_{\text {unsat }}^{*}\end{cases}
$$

where

$$
\begin{aligned}
& \rho_{\mathrm{o}, \text { unsat }}=(1-\beta) \rho_{\mathrm{o}, \mathrm{dry}}+\beta \rho_{\mathrm{o}, \mathrm{sat}} \\
& \rho_{\text {unsat }}^{*}=\left(1-\gamma_{1}\right) \rho_{\mathrm{dry}}^{*}+\gamma_{1} \rho_{\mathrm{sat}}^{\left(P=P_{\mathrm{Comp}}\right)} \\
& B_{\text {unsat,low }}=\frac{P_{\mathrm{Comp}}}{\left(\rho_{\text {unsat }}^{*}-\rho_{\mathrm{o}, \text { unsat }}\right)} \\
& B_{\text {unsat,high }}=\left[\frac{1}{\frac{(1-\beta)}{B_{\text {SolidComp }}}+\frac{\beta}{B_{\mathrm{Sat}}}}\right]
\end{aligned}
$$

where

$$
\gamma_{1}=\beta\left[\frac{1-\frac{P_{\text {Comp }}}{B_{\text {Sat }} \rho_{\text {sat }}^{*}}}{(1-\beta)\left(1-\frac{P_{\text {Comp }}}{B_{\text {Pl.Comp }} \rho_{\text {dry }}^{*}}\right)+\beta\left(1-\frac{\mathrm{P}_{\text {Comp }}}{\mathrm{B}_{\text {Sat }} \rho_{\text {sat }}^{*}}\right)}\right]
$$


Equation (15) reflects the fact that the compaction modulus of humid air residing in sand, consisting of dry air and water, is dominated by its more compliant phase (dry air).

In addition to specifying the pressure vs. density relation, the compaction model for sand entails the knowledge of the density dependence of the material's sound speed. The material sound speed is defined as a square-root of the ratio of the bulk modulus and the material mass density. The original compaction model for sand uses the following relation for the density-dependent bulk modulus for dry sand. The eq. (17) is obtained using a linear fit to the experimental bulk modulus vs. density data obtained in the work of Laine and Sandvik [20]:

$$
K_{\text {dry }}= \begin{cases}0 & \rho_{\text {dry }}<\rho_{\text {o,dry }} \\ -15.6302+0.0094074 \rho_{\text {dry }} & \rho_{\text {o,dry }}<\rho_{\text {dry }} \leqslant 0.8137 \rho_{s} \\ -93.05+0.0455 \rho_{\text {dry }} & 0.8137 \rho_{s} \leqslant \rho_{\text {dry }} \leqslant 0.9837 \rho_{s} \\ -1873.3+0.73074 \rho_{\text {dry }} & 0.9837 \rho_{\text {dry }}<\rho_{\text {dry }}<\rho_{s} \\ -3.233+0.022651 \rho_{\text {dry }}, & \rho_{\text {dry }}>\rho_{s}\end{cases}
$$

The density-dependent bulk modulus in saturated sand is derived following the same procedure as in the case of $P_{\text {sat }}$ vs. $\rho_{\text {sat }}$ relation as:

$$
K_{\text {sat }}=B_{\text {sat }} \rho_{\text {sat }}
$$

Likewise, the density-dependent bulk modulus for unsaturated sand is defined as:

$$
K_{\text {unsat }}\left(\rho_{\text {unsat }}, \alpha_{o}, \beta\right)=\left[\frac{1}{\frac{(1-\beta)}{K_{\mathrm{dry}}\left(\rho_{\mathrm{dry}}\right)}+\frac{\beta}{K_{\mathrm{sat}}\left(\rho_{\mathrm{sat}}\right)}}\right]
$$

where

$$
\rho_{\text {dry }}=\rho_{\text {unsat }}-\alpha_{o} \beta \rho_{w}
$$

and

$$
\rho_{\text {sat }}=\rho_{\text {unsat }}+\alpha_{o}(1-\beta) \rho_{w}
$$

As mentioned earlier, the density dependent sound speed (for dry, saturated and unsaturated sands) is defined as a square root of the ratio of the corresponding bulk moduli and mass densities.

Strength Model

As pointed out earlier, within the original compaction strength model for dry sand, the pressure dependence of yield stress is defined as:

$$
\sigma_{\mathrm{y}, \mathrm{dry}}=\mu_{1 \mathrm{dry}} P_{\mathrm{dry}} \approx \begin{cases}1.37 P_{\mathrm{dry}} & 0<P_{\mathrm{dry}} \leqslant P_{\mathrm{MC}} \\ 1.37 P_{\mathrm{MC}} & P_{\mathrm{dry}}>P_{M C}\end{cases}
$$

Also for the saturated sand, as discussed in our previous work [1], the pressure-dependent yield stress can be defined as:

$$
\sigma_{\mathrm{y}, \mathrm{sat}}= \begin{cases}\mu_{1 \text { sat }} P_{\mathrm{sat}} & 0 \leqslant P_{\mathrm{sat}} \leqslant P_{\mathrm{MC}} \\ \mu_{1 \text { sat }} P_{\mathrm{MC}} & P_{\mathrm{sat}}>P_{\mathrm{MC}}\end{cases}
$$

where the yield-stress-to-pressure proportionality coefficient, $\mu_{1 \text { sat }}$, is defined as:

$$
\mu_{1 \mathrm{sat}}= \begin{cases}0.1+1.275 \frac{P_{\mathrm{sat}}}{P_{\mathrm{MC}}} & 0 \leqslant P_{\mathrm{sat}} \leqslant P_{\mathrm{MC}} \\ 1.375 & P_{\mathrm{sat}}>P_{\mathrm{MC}}\end{cases}
$$

The term $P_{\mathrm{MC}}(=1.864 \mathrm{e} 5 \mathrm{kPa})$ appearing in Eqs (22)-(24) is the Mohr-Coulomb pressure beyond which the yield stress is pressure insensitive.

The yield stress vs. pressure relationship for the unsaturated sand can then be defined using a linear combination of the yield-stress/pressure proportionality coefficients in dry and the saturated sands as:

$$
\sigma_{\mathrm{y}, \text { sat }}=\left\{\begin{array}{l}
\mu_{1 \text { un sat }} P_{\text {sat }} 0 \leqslant P_{\text {unsat }} \leqslant P_{\mathrm{MC}} \\
\mu_{\text {1un sat }} P_{\mathrm{MC}} P_{\text {unsat }}>P_{M C}
\end{array}\right.
$$


Table 2

Coordinates of the pressure transducers located in air. The origin of the coordinate system is located along the line of symmetry at the sand/air interface

\begin{tabular}{lcc}
\hline Transducer designation & \multicolumn{2}{c}{ Transducer coordinates, cm } \\
\cline { 2 - 3 } & $\mathrm{X}$ & $\mathrm{Y}$ \\
\hline PT_30_0 & -30.00 & 0 \\
PT_30_22.5 & -27.71 & 11.48 \\
PT_30_45 & -21.21 & 21.21 \\
PT_70_0 & -70.00 & 0 \\
PT_70_30 & -60.62 & 35.00 \\
PT_110_0 & -110.00 & 0 \\
\hline
\end{tabular}

where

$$
\mu_{1 \text { unsat }}=(1-\beta) \mu_{1 \mathrm{dry}}+\beta \mu_{1 \mathrm{sat}}
$$

In addition to specifying the yield stress vs. pressure relationship, the compaction strength model entails the knowledge of the density dependent shear modulus. Since water has no ability to support shear stresses, the shear modulus, $G$, of unsaturated sand is dominated by the shear modulus of the solid skeleton of the sand. However, the presence of water changes the density of the sand. Therefore, the original compaction shear modulus vs. density relationship defined (using ten pairs of $(\mathrm{G}, \rho)$ points in AUTODYN, Fig. 3(d)) has to be modified by adding a term $\alpha \beta \rho_{w}$ to the values of density in order to obtain a (deformation-rate independent) shear modulus vs. density relationship for unsaturated sand.

\section{Failure Model}

It is well established that the presence of moisture in sand increases the sand's cohesive strength [22]. Therefore, the magnitude of the (negative) failure pressure for sand is expected to increase with the saturation ratio $(\beta)$. Also, the moisture content should be substantial $(\beta>0.7)$ before its effect on the cohesive strength of sand becomes significant [22]. The following expression is proposed for the magnitude of the (negative) failure pressure in unsaturated sand; $P_{\text {fail, unsat }}$ :

$$
P_{\text {fail, unsat }}=\beta^{5} P_{\text {fail, sat }}
$$

where $P_{\text {fail,sat }}$ (set equal to $729 \mathrm{kPa}$ ) is the failure pressure in saturated sand [22]. Equation (27) is a purely phenomenological relation and was selected as a single-term high-order polynomial which best satisfied the condition that the cohesive strength of unsaturated sand with a saturation ratio of 0.7 is around $10-15 \%$ of that in the saturated sand.

The effect of degree of saturation on sand behavior

To reveal the effect of saturation ratio (degree of saturation) on the behavior of sand as predicted by the simplified compaction material model presented in the present work; P vs. $\rho$, C vs. $\rho, \sigma_{y}$ vs. $\rho, \mathrm{G}$ vs. $\rho$ and $P_{\text {fail }}$ vs. $\beta$ at three degrees of saturation $(0,0.5$, and 1$)$ are displayed respectively in Fig. 3(a)-(e). The initial porosity of sand is $\alpha_{0}=$ 0.36 .

\subsection{Problem definition}

The problem analyzed computationally in the present study is identical to the one investigated experimentally in Ref. [2]. A schematic of the problem is shown in Fig. 4. Due to the inherent axial symmetry of the set-up used in Ref. [2], the mine detonation is analyzed as a $2 \mathrm{D}$ axi-symmetric problem. The problem can be briefly described as follows:

A $1.27 \mathrm{~cm}$ wall thickness cylindrical barrel with the outer-diameter of $81.6 \mathrm{~cm}$ and the overall height of $71 \mathrm{~cm}$ is filled with sand up to its top. A $100 \mathrm{~g}$ cylindrical-disk shape $\mathrm{C} 4$ high-energy explosive $(6.4 \mathrm{~cm}$ in diameter and $2 \mathrm{~cm}$ in height) is buried into the sand along the centerline of the barrel with its faces parallel with the sand surface. The Depth of Burial (DOB) (defined as the vertical distance between the top face of the explosive and the sand surface) is varied in a range between 0 and $8 \mathrm{~cm}$. Thus a $0 \mathrm{~cm}$ DOB case corresponds to a flush-buried explosive. A set of six pressure transducers is utilized to monitor the pressure in the air following the detonation of the explosive. 

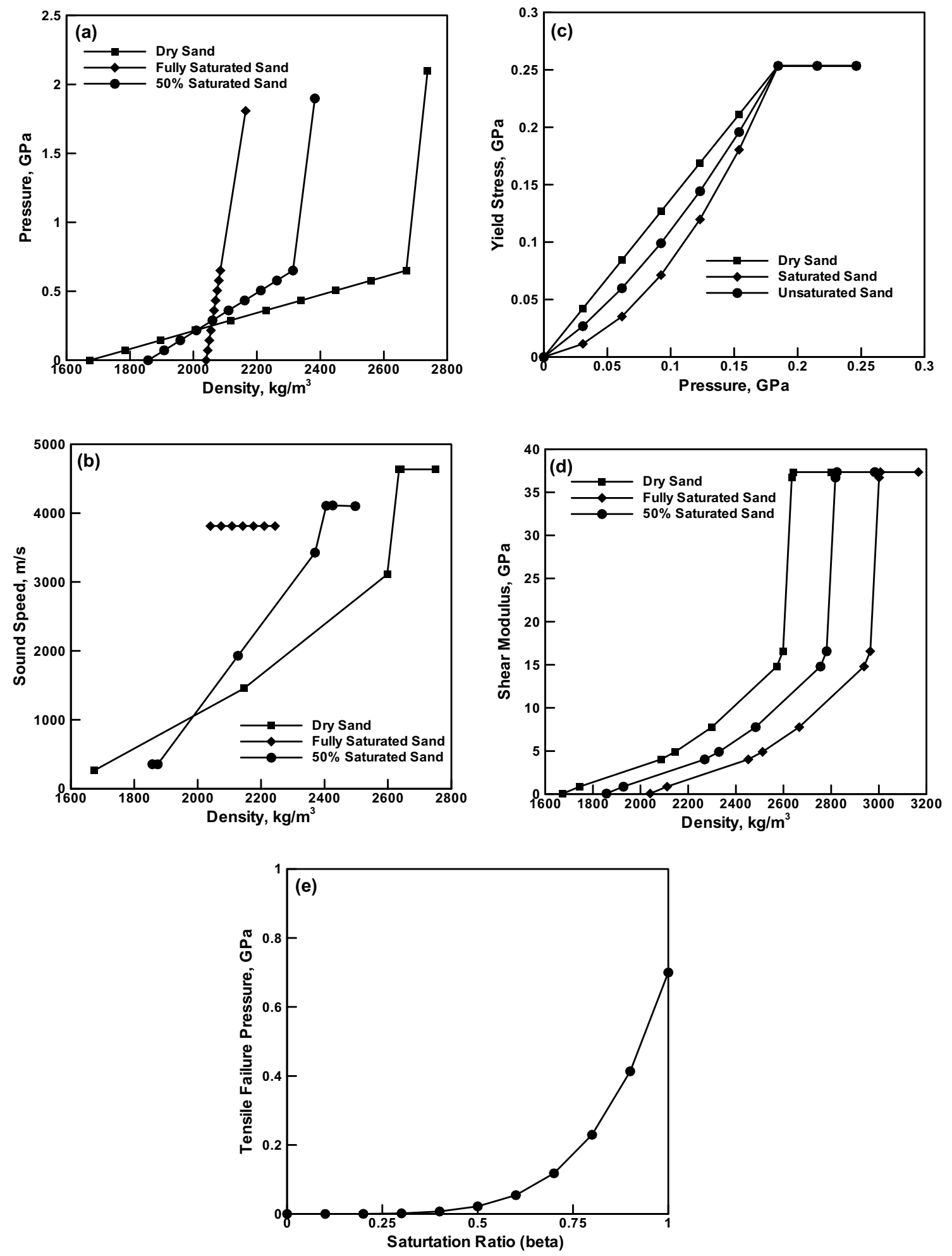

Fig. 3. Effect of the saturation ratio on the (a) pressure vs. density; (b) sound speed vs. density; (c) yield stress vs. pressure; (d) Shear modulus vs. density and (e) the magnitude of the negative failure pressure. 


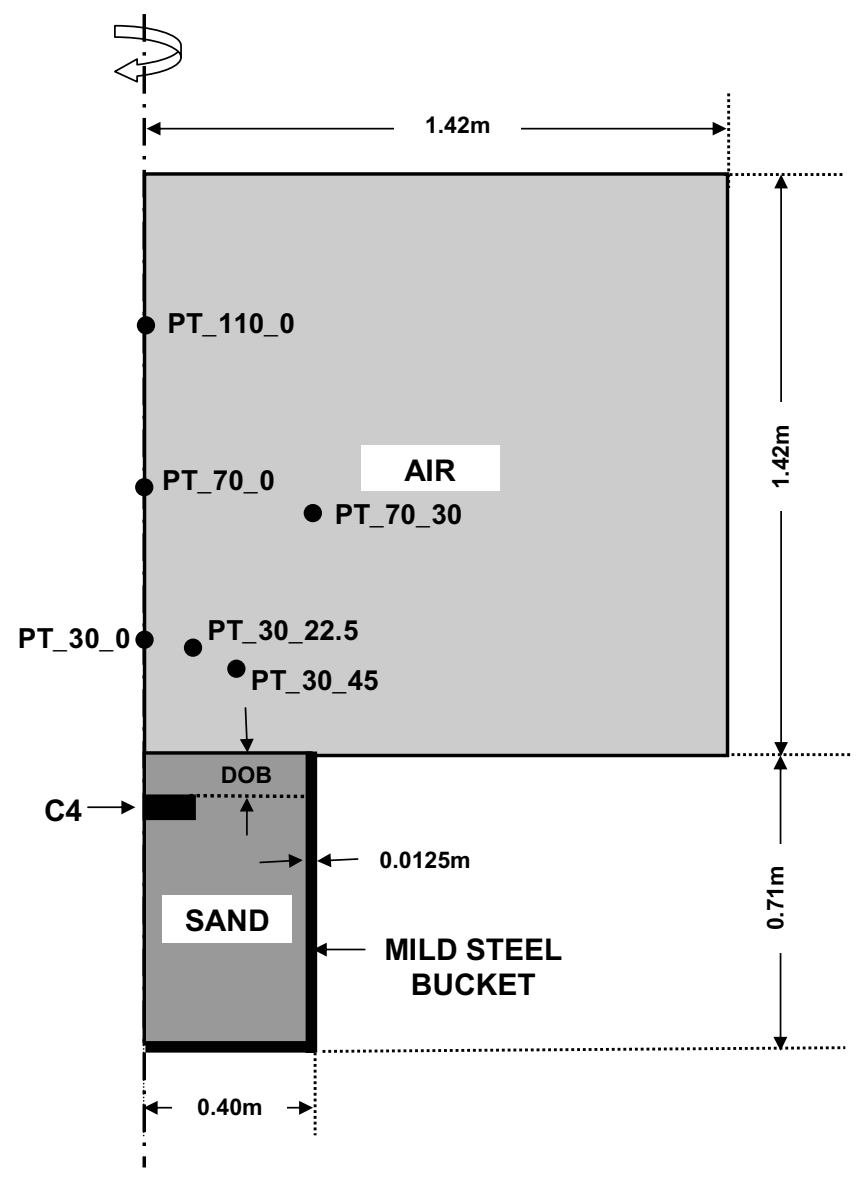

Fig. 4. A simple schematic of the experimental setup used in Ref. [2] to study the effect of explosion of a shallow-buried mine.

The designations and the position coordinates of the six transducers are given in Table 2. The first number in the Pressure Transducer (PT) designation represents the distance in centimeters of the transducer from the origin of the coordinate system (defined below), while the second number represents the angular relation in degrees between the position vector of the pressure transducer and the axis of symmetry. The location of the six pressure transducers is also shown in Fig. 4. To be consistent with the definition of coordinate system for the 2D axi-symmetric problem used in AUTODYN [10], the $y$ coordinates are measured in the radial direction from the centerline of the barrel, while the $x$ coordinates are measured along the axis of symmetry, with $x=0$ corresponding to the sand surface and $x<0$ denoting the air region above the ground.

The physical model of the experimental setup used in Ref. [2] is displayed in Fig. 4. The corresponding computational multi-material Euler model is shown in Fig. 5. In Fig. 5, various portions of the computational domain are filled (as indicated in the figure) with one or more of the attendant materials (air, sand, C4 gaseous-detonation products and AISI 1006 mild steel). The topmost portion of the computational domain is filled with air and the locations of the pressure transducers located in air are denoted as dots. Table 3 provides a list of the transducers and their coordinates. The domain just below the air domain consists of sand and steel bucket enclosure. The left boundary in Fig. 5 coincides with the axis of symmetry ( $x$-axis). The horizontal direction ( $y$-axis) corresponds to the radial direction.

The "flow-out" boundary conditions are applied to all the outer boundaries of the air-filled portion of the computational domain. The "flow-outf" boundary condition allows the simulation of a free air blast by allowing the air to escape the computational Euler domain with the propagation of blast gases and sand following detonation. "No-flow" boundary conditions are applied to the outer boundaries associated with the steel bucket. It should be noted that due to the use of a multi-material Euler processor, no boundary conditions have to be defined for the sand/steel and 
Table 3

Material Models used for Air, AISI 1006 Steel and C4 used in the present work. Model parameters can be referenced from AUTODYN materials library [10]

\begin{tabular}{|c|c|c|c|}
\hline Material & Equation of state & Strength model & $\begin{array}{l}\text { Failure } \\
\text { model }\end{array}$ \\
\hline Air & $\begin{array}{c}\text { Ideal Gas [10] } \\
P=(\gamma-1) \frac{\rho}{\rho_{o}} E\end{array}$ & None & None \\
\hline AISI & Linear & Johnson-Cook & None \\
\hline $\begin{array}{l}1006 \\
\text { Steel }\end{array}$ & $P=K \mu$ & $Y=\left[A+B_{1} \varepsilon_{p l}^{n}\right]\left[1+C_{1} \log \dot{\varepsilon}_{p l}\right]\left[1-T_{H}^{m}\right]$ & \\
\hline $\mathrm{C} 4$ & $\begin{array}{c}\text { Jones-Wilkins-Lee [17] } \\
P=A_{1}\left(1-\frac{w}{R_{1} v}\right) e^{-R_{1} v}+B_{2}\left(1-\frac{w}{R_{2} v}\right) e^{-R_{2} v}+\frac{w E}{v}\end{array}$ & None & None \\
\hline
\end{tabular}

sand/air interfaces. The constitutive equations pertaining to the response of the four materials to a (hydrodynamic) pressure, a deviatoric stress and/or a negative pressure have been previously discussed in Section 2.2.

To mimic the detonation initiation conditions used in Ref. [2], detonation is initiated at the central circular portion of the explosive of radius $3.2 \mathrm{~cm}$, at the bottom face of the mine. To monitor the temporal evolution of pressure in air, six gage points are introduced whose locations coincide with those of the pressure transducers used in Ref. [2].

\section{Results and discussion}

\subsection{The response of surrounding soil to the explosion of a shallow-buried mine}

Before the results obtained in the present study are presented and discussed, a brief overview of the phenomena accompanying an explosion of a shallow-buried mine is presented. These phenomena have been observed experimentally (e.g. [7]) and computationally (e.g. [1]). While an explosion is a continuous event taking place over a relatively short period of time (typically several hundred microseconds in duration), it is often considered to involve three distinct phases. A brief discussion of the three phases of landmine explosion is discussed in this section.

In the first stage of landmine explosion, shock waves are generated and begin to propagate from the points of initiation of the explosion, transforming an (typically solid) explosive into a mass of hot, high-pressure gaseous detonation products. The interactions of the high-pressure detonation products with the surrounding sand result in different responses of different portions of the sand depending (primarily) on their distance from the explosive and on their physico-mechanical properties. In the innermost sand zone, commonly referred to as the "zone of crushing", which extends to 2-3 effective explosive radii from the explosive surface, the pressure and the temperature experienced by the sand are so high that the shock-wave energy transmission to the sand is essentially independent of the structure or physico-mechanical properties of the sand. Within this zone, the sand is fully compacted and/or, to a great extent, vaporized. In the second zone, commonly referred to as the "zone of plastic deformation", which extends 3-6 effective explosive radii from the explosive surface, irreversible crushing and compaction of the sand takes place. In the zone which is furthest away from the explosive, the "zone of elastic deformation", the shock-wave energy transmission results only in a reversible (elastic) deformation of the sand.

The initial stage of explosion plays an important role in the overall effectiveness and lethality of a buried mine since it controls the amount of explosive energy available to impact the target structure/personnel. Many parameters affect the effectiveness of a landmine, among which, the most important are the depth of burial, sand physical and mechanical properties and the moisture content $[1,3,5]$. The optimal depth of burial for shallow buried explosives corresponds to a condition under which the charge explosion is followed by a preferential venting of the detonation products and sand ejection in the upward direction while the amount of the explosion energy absorbed by the (un-ejected) sand is minimized. Lower porosity levels and larger moisture contents generally give rise to an increase in the sand's ability to transmit shock and reduce sand's ability to absorb energy.

In the second stage of the landmine explosion, which is dominated by the expansion of gaseous detonation products, compression waves initiated at the detonation products/sand interface travel through the sand and reach the sand/air interface where they are partially reflected as a tensile pressure waves and partially transmitted to the air as 


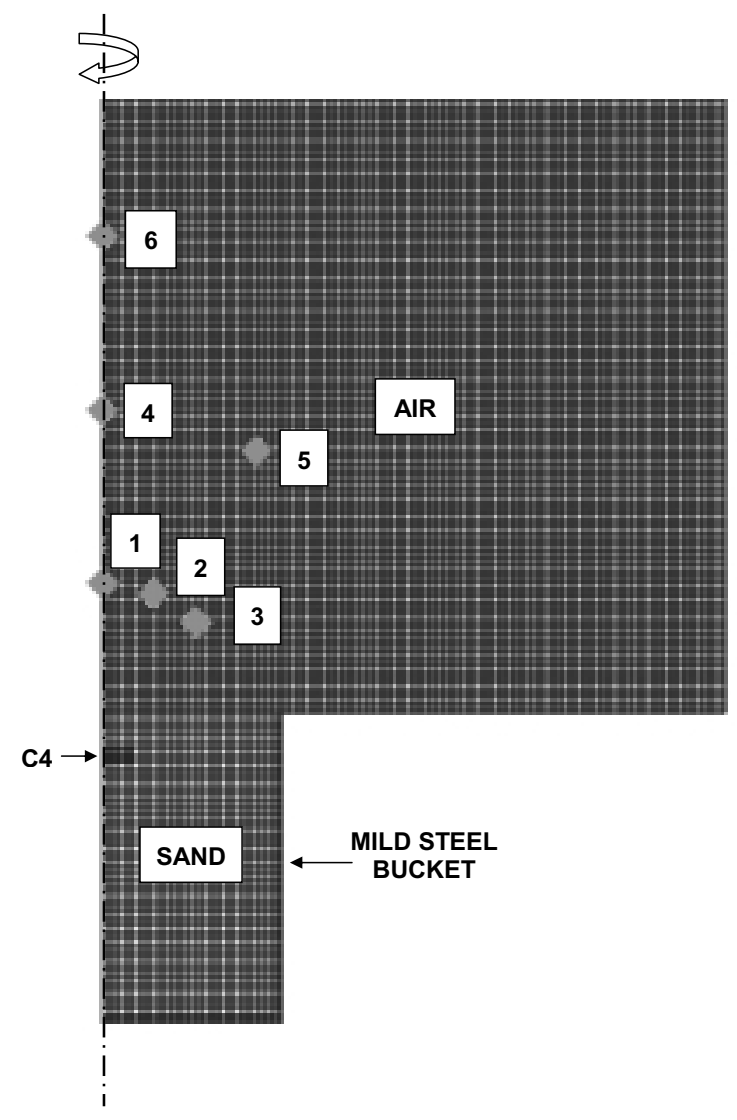

Fig. 5. Computational sub-domains used in the present work.

blast waves. The expansion of the detonation products gives rise to the formation and growth of an overburden sand bubble. However, the aforementioned tensile waves cause fragmentation of the sand overburden and the resulting sand fragments, under the influence of high-pressure detonation products, become ejected upward creating a cavity in the ground. The high pressure/high temperature detonations products residing in the cavity continue to expand (adiabatically) leading to the formation of additional blast waves in air. These waves are capable of transferring large amount of energy to the target, while rarefaction waves are generated within the detonation products.

In the third stage of explosion, complex interactions between the compression waves and the rarefaction waves in the detonation products and the sand within the cavity give rise to the erosion and ejection of the surrounding sand leaving a crater in the ground whose volume is $2-3$ orders of magnitude larger that that of the initial landmine.

Typical results obtained in the present work that clearly outline the effect of the degree of saturation of sand on the temporal evolution of the material distribution following detonation of a shallow-buried mine are displayed in Figs 6 and 7. The results shown in Fig. 6 pertain to the case of perfectly dry sand while the results shown in Fig. 7 correspond to that of fully saturated sand. In general, two main effects of the degree of saturation are observed:

(a) A substantially larger height of the sand over-burden bubble is obtained in the case of saturated sand before the bubble ruptures and the venting of detonation gases takes place. This finding is consistent with the fact that due to a lubrication effect of water in sand, the strength of saturated sand is reduced relative to that of dry sand. At the same time, increased adhesion strength of saturated sand gives rise to a delay in its fracture; and

(b) Venting of the detonation products and ejection of sand is directed more in a direction normal to the air/sand overburden interface in the case of saturated sand. 

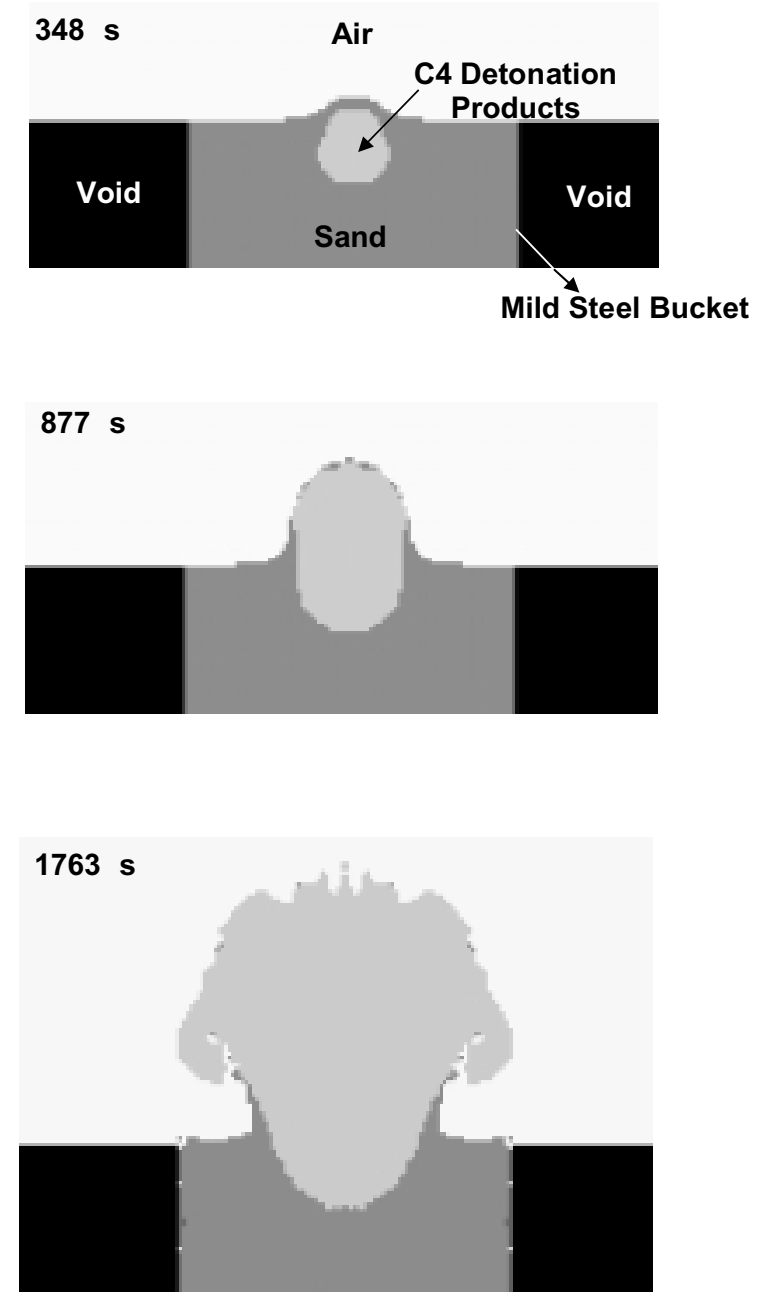

Fig. 6. Temporal evolution of material deformation in the case of landmine detonation (DOB $=8 \mathrm{~cm}$ ) in the case of dry sand.

\subsection{Landmine detonation in "dry” sand}

The ability of the present material model for sand to account for the main observations obtained during experimental investigation of landmine detonation in "dry" (50wt\%-sand/50wt\%-clay) soil (average degree of saturation $\beta=0.15$ ) [2] is discussed in this section. A comparison of the computational and experimental results pertaining to the stand-off distance (distance between the pressure transducer and the air/sand interface) dependencies of the peak over-pressure and the time of blast-wave arrival (along the axis of symmetry), the angular dependence of the time of arrival and the temporal evolution of the sand-overburden bubble height are shown respectively in Figs 8(a)-(d). The results displayed in Figs 8(a)-(d) can be summarized as follows:

(a) At a given value of the stand-off distance, the peak side-on (static) overpressure (the difference between the absolute pressure and the atmospheric pressure) decreases as the DOB increases, Fig. 8(a). This is the result of the fact that as the thickness of the sand overburden increases a larger fraction of the potential energy contained within the high-pressure detonation products is absorbed by the compacting sand. Furthermore, at a given DOB, the peak over-pressure decreases with an increase with the transducer distance from the air/sand interface as a result of various blast-wave attenuation and dispersion processes. The agreement between the computational results and their experimental counterparts is reasonable in the case of $3 \mathrm{~cm}$ and $8 \mathrm{~cm}$ DOBs. However, the agreement is only fair in the case of $0 \mathrm{~cm}$ DOB. This may not be solely the result of the potential 

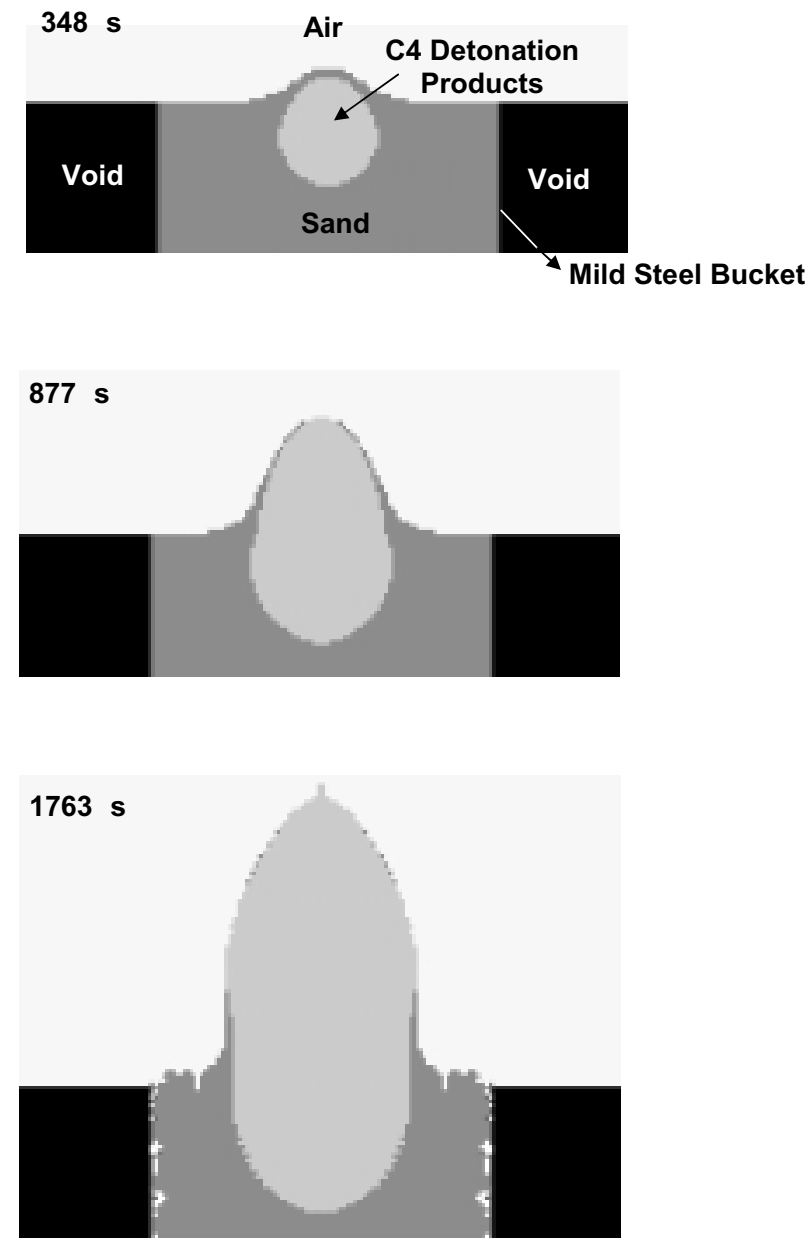

Fig. 7. Temporal evolution of material deformation in the case of landmine detonation (DOB $=8 \mathrm{~cm}$ ) in the case of fully saturated sand.

deficiencies of the present material model for sand since the role of sand is least critical in the case of a flush-buried mines;

(b) At a given value of the stand-off distance, the time of arrival of the blast waves increases with an increase in DOB, Fig. 8(b). This is the result of the fact that as the DOB increases, the distance between a given pressure transducer and the explosive also increases. For the same reason, at a fixed value of DOB, the time of arrival increases with an increase in the transducer distance from the air/sand interface. At the DOBs of $3 \mathrm{~cm}$ and $8 \mathrm{~cm}$, the agreement between the computational and experimental results is reasonable. On the other hand at $0 \mathrm{~cm}$ DOB the agreement is less satisfactory. This may be, at least partly, caused by the fact that due to the large magnitude of blast pressures in the case of flush-buried mine, transducer signals contained a lot of noise making precise estimation of the arrival time (as well as peak pressures) quite difficult;

(c) At a given value of DOB, the time of arrival of the blast waves is quite insensitive to the offset angle of the transducer from the symmetry axis, Fig. 8(c). This could be explained by the fact that while the offset angle is varied, the offset distance (the distance between the top center point of the mine and the transducer) was kept constant. At each of the three values of the DOB, the agreement between the computational and experimental results is reasonable; and

(d) The agreement between the computational and the experimental results pertaining to the temporal evolution of sand bubble height is very good in the case of $8 \mathrm{~cm}$ DOB, Fig. 8(d). In the case of $3 \mathrm{~cm}$ DOB the agreement is also good but caution should be exercised from drawing conclusions due to a limited number of experimental 

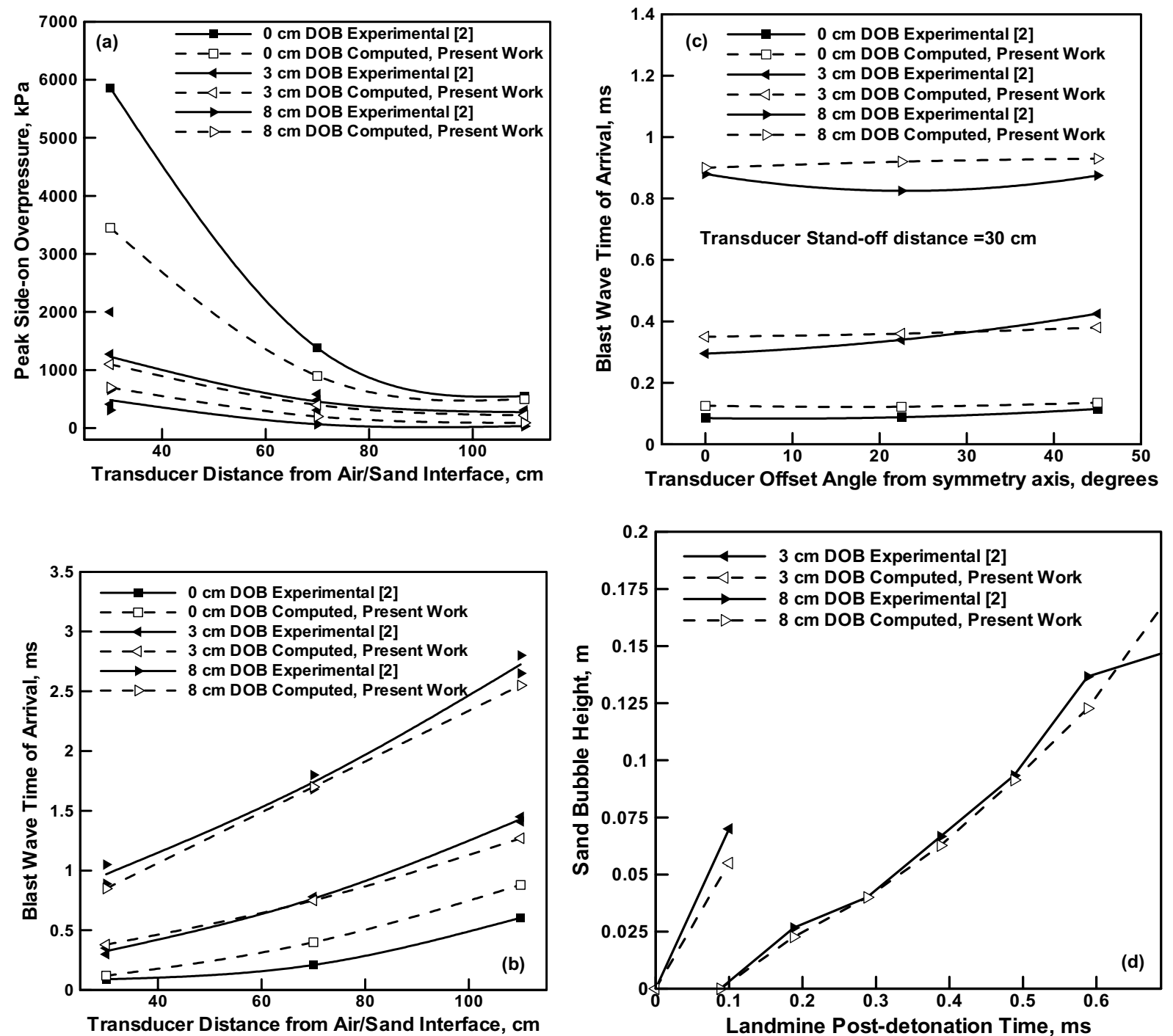

Fig. 8. A comparison of the experimental [2] and computed (present work) results pertaining to various phenomena associated with landmine detonation in dry sand: (a) Side-on overpressure vs. transducer distance from air/sand interface; (b) Blast wave arrival time vs. transducer distance from air/sand interface; (c) Blast wave arrival time vs. transducer offset angle from the symmetry axis and (d) Sand bubble height vs. landmine post-detonation time.

data points. Further, it should be noted that due to the absence of sand-overburden in the case of 0cm DOB, no sand bubble is formed during landmine detonation.

Based on the aforementioned findings, it appears that the present material model for sand can account reasonably well for the ballistic behavior of dry soil containing a substantial fraction of clay. This conclusion is in agreement with the experimental observations reported in Ref. [3] which showed that the soil type (e.g. prairie sand containing high level of silt and clay vs. impurity-free $-30 /+50$ sand) has a second-order effect on the ballistic behavior of the soil.

\section{Landmine detonation in fully saturated sand}

The ability of the present material model for sand to account for the main observations obtained during experimental 
investigation of landmine detonation in fully saturated (50wt\%-sand $/ 50 \mathrm{wt} . \%$-clay) soil (average degree of saturation $\beta \sim 1.0$ ) [2] is discussed in this section. In general, the presence of clay in sand is expected to change the materials equation of state (by altering its compaction behavior and/or its bulk modulus/speed of sound) as well as the material's strength and failure constituent responses (wet clay mixed with sand acts as a binder increasing the materials shear strength and cohesive strength). However, the extent of the effect of clay on the ballistic response of saturated sand is not well established. A comparison of the computational and experimental results pertaining to the stand-off distance dependencies of the peak over-pressure and the time of blast-wave arrival (along the axis of symmetry), the angular dependence of the time of arrival and the temporal evolution of the sand-overburden bubble height are shown respectively in Figs 9(a)-(d). The agreement between the computational results and their experimental counterparts displayed in Figs 9(a)-(d) can be summarized as follows:

(a) In the case of peak overpressure vs. transducer distance from air/sand interface results, the agreement is reasonable in the case of $3 \mathrm{~cm}$ and $8 \mathrm{~cm}$ DOBs. In the case of $0 \mathrm{~cm}$ DOB (the case associated with the largest uncertainty in the experimental results),on the other hand, the agreement is only fair. It should be also pointed out that in the case of $0 \mathrm{~cm} \mathrm{DOB}$ and $30 \mathrm{~cm}$ stand-off distance, the observed good agreement is most likely fortuitous. This conjecture is based on two observations: (i) the transducer in question showed visible signs of mechanical wear and damage, at the time when mine-detonation experiments in saturated soil were carried out [2] and (ii) the experimental values for the pressure $(\sim 4000 \mathrm{kPa})$, in the case of saturated soil are inexplicably substantially lower than its counterpart in dry soil $(\sim 6000 \mathrm{kPa})$;

(b) In the case of the time of arrival vs. stand-off distance results the agreement is reasonable for all three values of DOB;

(c) Both the experimental and the computational results show that the time of arrival of the blast waves increases with an increase in the offset angle of the transducer from the symmetry axis, Fig. 9(c). However, the quantitative agreement between the two sets of results is only fair at each of the three DOBs; and

(d) In the case of the height of sand bubble vs. landmine post-detonation time results, Fig. 9(d), the agreement between the computational and the experimental results is only fair. Since the computational results consistently under predict the measured sand bubble heights, it appears that the presence of clay reduces the soil strength, the effect which is not accounted for in the present material model for sand.

The results presented in this section and the previous section suggest that the present material model for sand when used in conjunction with the appropriate transient non-linear dynamics simulations can reasonably well account for the magnitude, spatial distribution and temporal evolution of the dynamic loads accompanying detonation of shallowburied mines in soil with various levels of clay and water contents. In the case of detonation of flush-buried mines, the effect of the material model for sand is expected to be less pronounced and, hence, less critical. Consequently, the observed discrepancies between the experimental and the computational results in the case of $0 \mathrm{~cm}$ DOB can not be readily interpreted as the shortcomings of the sand material model. This conclusion is further supported by the fact that the corresponding experimental results were associated with substantial uncertainty (typically the standard deviation was $25-30 \%$ of the mean value). Also, there are additional phenomena which were not accounted for in the transient non-linear dynamics analysis of the mine detonation. For instance, dynamic pressure, in addition to the static pressure, can contribute to the transducer signal; evaporated water can significantly alter the properties of air, etc. It is interesting to note that the two aforementioned phenomena are expected to be most prevalent in the case of $0 \mathrm{~cm}$ DOB and the saturated soil, the case associated with the largest mismatch between the experimental and the computational results.

\section{Summary and conclusions}

Based on the results obtained in the present work, the following main summary remarks and conclusions can be drawn:

1. Our recently developed compaction material model for sand [1] has bean simplified by eliminating explicit strain-rate dependences of its parameters and upgraded by including a degree of saturation dependence of the (minimum) failure pressure. 

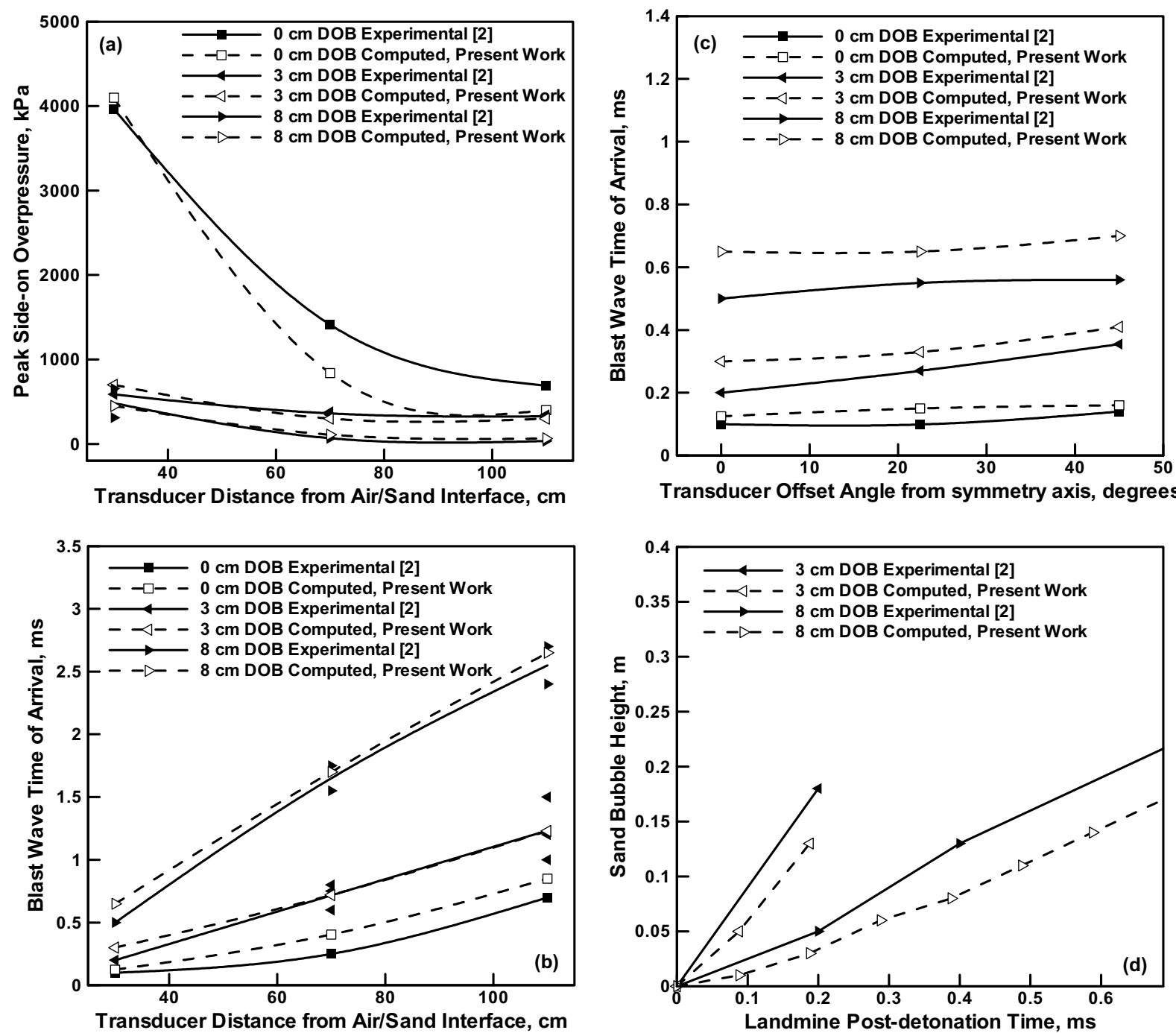

Fig. 9. A comparison of the experimental [2] and computed (present work) results pertaining to various phenomena associated with landmine detonation in fully saturated sand: (a) Side-on overpressure vs. transducer distance from air/sand interface; (b) Blast wave arrival time vs. transducer distance from air/sand interface; (c) Blast wave arrival time vs. transducer offset angle from the symmetry axis and (d) Sand bubble height vs. landmine post-detonation time.

2. The extent of agreement between the computational and experimental results obtained suggests that the present material model for sand can account reasonably well for the ballistic behavior of dry soil containing substantial amounts of clay.

3. In the case of saturated soil, the agreement between the computational and experimental results is less satisfactory. Since the discrepancies are most pronounced in the case of flush-buried mines where the role of the soil material model is least critical, it is argued that the limitations of the experimental technique used and a high level of moisture in the air above the explosion site are, at least partly, responsible for the observed mismatch between the computational and the experimental results.

\section{Acknowledgements}

The material presented in this paper is based on work supported by the US Army/Clemson University Cooperative 
Agreement W911NF-04-2-0024 and by the US Army Grant Number DAAD19-01-1-0661. The authors are indebted to Dr. Fred Stanton for the support and a continuing interest in the present work.

\section{References}

[1] M. Grujicic, B. Pandurangan and B. Cheeseman, The effect of degree of saturation of sand on detonation phenomena associated with shallow-buried and ground-laid mines, Journal of Shock and Vibration 13 (2006), 41-61.

[2] J. Foedinger, Methodology for Improved Characterization of Landmine Explosions, SBIR Phase-II Plus Program, Technical Interchange Meeting, Material Science Corporation, June 2005.

[3] D. Bergeron and J.E. Trembley, Canadian Research to Characterize Mine Blast Output, $16^{\text {th }}$ International MABS Symposium, Oxford, UK, September 2000.

[4] K.A. Holsapple and K.R. Housen, Crater Database and Scaling Tools, http://keith.aa.washinton.edu/craterdata, November 2004.

[5] P.S. Westine, B.L. Morris, P.A. Cox and E. Polch, Development of Computer Program for Floor Plate Response from Landmine Explosions, Contract Report No. 1345, for US Army TACOM Research and Development Center, 1985.

[6] B.L. Morris, Analysis of Improved Crew Survivability in Light Vehicles Subjected to Mine Blast, Final Report for Contract No. DAAK7092-C-0058 for the US Army Belvoir RDEC, Ft. Belvoir, VA, 1993.

[7] D. Bergeron, S. Hlady and M.P. Braid, Pendulum Techniques to Measure Landmine Blast Loading, 1th International MABS Symposium, Las Vegas, USA, June 2002.

[8] M.P. Braid, Experimental Investigation and Analysis of the Effects of Anti-personnel Landmine Blasts, Defence R\&D Canada, Suffield Special Publication, DRES SSSP 2001-188, December 2001.

[9] M. Grujicic, B. Pandurangan and B. Cheeseman, A Computational Analysis of Detonation Phenomena Associated with Mines Shallowburied in Sand, Multidiscipline Modeling in Materials and Structures, accepted for publication, January 2006.

[10] AUTODYN- 2D and 3D, Version 6.1, User Documentation, Century Dynamics Inc., 2006.

[11] D. Bergeron, R. Walker and C. Coffey, Detonation of 100-gram Anti-Personnel Mine Surrogate Charges in Sand-A Test Case for Computer Code Validation, Suffield Report No. 668, Defence Research Establishment Suffield, Ralston, Alberta, Canada, April 1998.

[12] M. Grujicic, B. Pandurangan and B. Cheeseman, Impulse loading resulting from landmines buried in water saturated sand, Journal of Materials: Design and Applications, submitted for publication, March 2006.

[13] L.C. Taylor, R.R. Skaggs and W. Gault, Vertical Impulse Measurements of Mines Buried in Saturated Sand, Fragblast, 9, n 1, March 2005, 19-28.

[14] D.L. Youngs, Time Dependent Multi-material Flow with Large Flow Distortion, Numerical Methods for Fluid Dynamics, Academic Press, 1982.

[15] D. Schwer and K. Kailasanath, Blast Mitigation by Water Mist (1) Simulation of Confined Blast Waves, Report NRL/MR/6410-02-8636, Naval Research Laboratory, Washington, August 2002.

[16] J. Henrych, The Dynamics of Explosion and Its Use, Chapter 5, Elsevier Publications, New York, USA, 1979.

[17] E.L. Lee, H.C. Hornig and J.W. Kury, Adiabatic Expansion of High Explosive Detonation Products, UCRL - 50422, Lawrence Radiation Laboratory, University of California, 1968.

[18] Z. Wang, H. Hao and Y. Lu, A Three-phase Soil Model for Simulating Stress Wave Propagation due to Blast Loading, International Journal for Numerical and Analytical methods in Geomechanics 28 (2004), 33-56.

[19] B. Loret and N. Khalili, A three-phase Model for Unsaturated Soils, International Journal for Numerical and Analytical Methods in Geomechanics 24 (2000), 893-927.

[20] P. Laine and A. Sandvik, Derivation of Mechanical Properties for Sand, Proceedings of the 4th Asia-Pacific Conference on Shock and Impact Loads on Structures, CI-Premier PTE LTD, Singapore, November 2001, 361-368.

[21] D. Dowson and P. Ehret, Past, Present and Future Studies in Elastohydrodynamics, Proc Instn Mech Engrs 213(part J) (1999), 317-333.

[22] S. Stein and T. Kim, Effect of moisture on attraction force on beach sand, Marine Geosources and Geotechnology 22 (2004) $33-47$.

[23] J.G. Jackson, J.Q. Ehrgott and B. Rohani, Loading rate effects on compressibility of sand, ASCE Journal of Geotechnical Engineering Division 106 (August 1980), 839-885.

[24] D.J. Chapman, K. Tsembelis and W.G. Proud, The Behavior of Water Saturated Sand Under Shock-loading, American Physical Society, 14th APS Topical Conference on Shock Compression of Condensed Matter, July, 2005. 

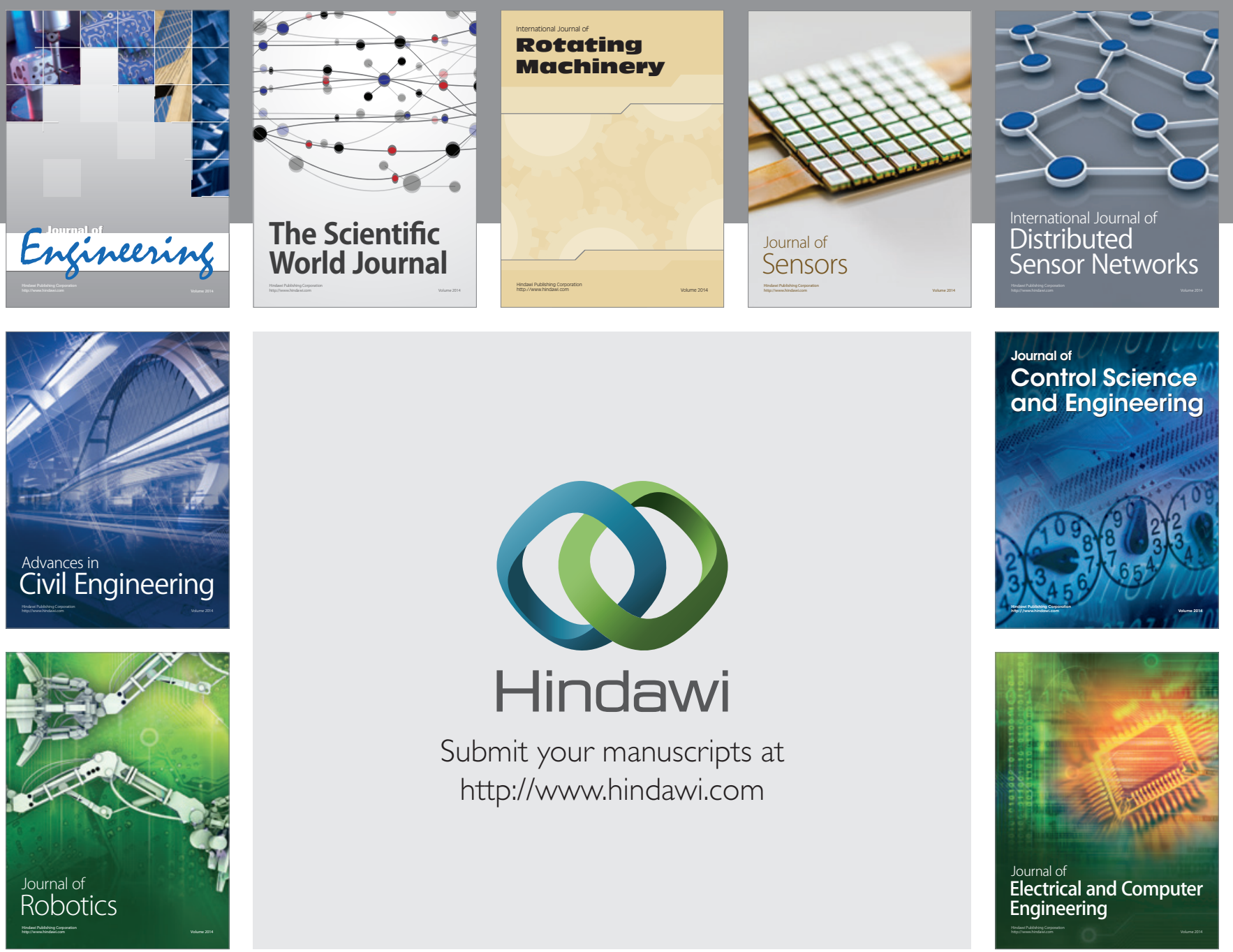

Submit your manuscripts at

http://www.hindawi.com
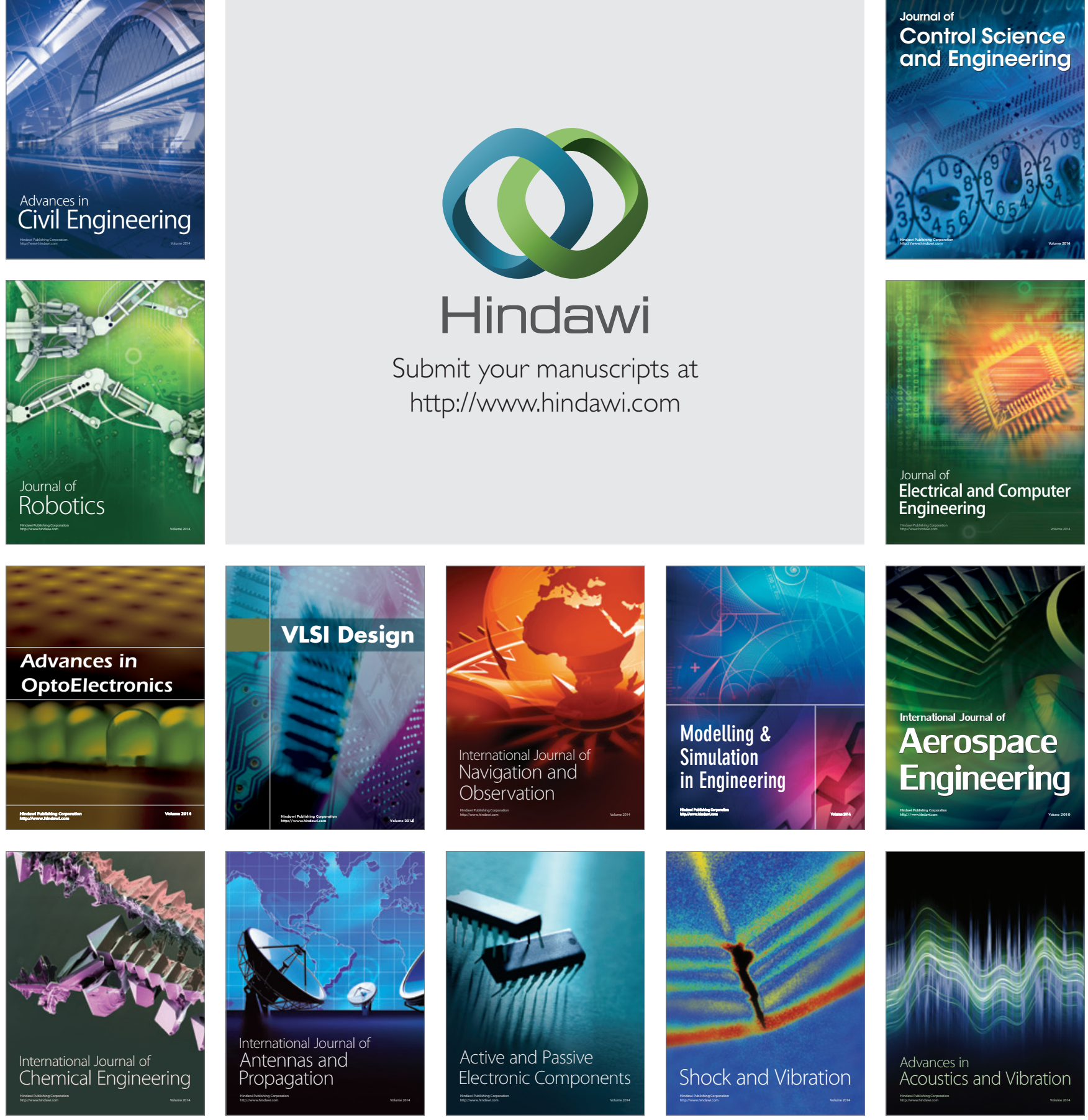